EPJ Web of Conferences 79, 01005 (2014)

DOI: $10.1051 /$ epjconf/20147901005

(C) Owned by the authors, published by EDP Sciences, 2014

\title{
Production of solar fuels by $\mathrm{CO}_{2}$ plasmolysis
}

Adelbert P.H. Goede ${ }^{1, a}$, Waldo A. Bongers ${ }^{1}$, Martijn F. Graswinckel ${ }^{1}$, Richard M.C.M van de Sanden ${ }^{1}$, Martina Leins ${ }^{2}$, Jochen Kopecki ${ }^{2}$, Andreas Schulz ${ }^{2}$ and Mathias Walker ${ }^{2}$

${ }^{1}$ Dutch Institute for Fundamental Energy Research, PO Box 1207, 3430 BE Nieuwegein, The Netherlands

${ }^{2}$ Institute of Interfacial Process Engineering and Plasma Technology, 70569 Stuttgart, Germany

\begin{abstract}
A storage scheme for Renewable Energy (RE) based on the plasmolysis of $\mathrm{CO}_{2}$ into $\mathrm{CO}$ and $\mathrm{O}_{2}$ has been experimentally investigated, demonstrating high energy efficiency $(>50 \%)$ combined with high energy density, rapid start-stop and no use of scarce materials. The key parameter controlling energy efficiency has been identified as the reduced electric field. Basic plasma parameters including density and temperature are derived from a simple particle and energy balance model, allowing parameter specification of an upscale $100 \mathrm{~kW}$ reactor. With RE powered plasmolysis as the critical element, a $\mathrm{CO}_{2}$ neutral energy system becomes feasible when complemented by effective capture of $\mathrm{CO}_{2}$ at the input and separation of $\mathrm{CO}$ from the output gas stream followed by downstream chemical processing into hydrocarbon fuels.
\end{abstract}

\section{Renewable energy and the need for storage}

While Renewable Energy (RE) sources including solar photo-voltaic, concentrated solar and wind make up an increasingly large fraction of the EU energy supply mix, the development of a suitable energy storage scheme has lagged behind. The need for storage is evident from the ill-matched supply and demand characteristics of RE both geographically and temporarily. This already causes problems in transport and uptake of RE, in particular the handling of excess wind power by the electricity grid [1]. In order to meet base load demand the presently installed wind power capacity is vastly under-employed whilst peak power handling requires over capacity on the grid, driving up total cost of the RE system. Storage capacity needed at EU level to bridge one day of electrical power demand is of the order of $10 \mathrm{TWh}$. By comparison, EU hydropower storage capacity is approximately $15 \mathrm{TWh}$. The only way to meet EU energy storage requirements over several days would be chemical storage [2].

In order to meet the EU 2020 energy and climate change targets and to follow the EU 2050 Energy Roadmap, it is necessary to reduce $\mathrm{CO}_{2}$ emission and to move away from fossil fuel altogether. However, a RE driven system could still employ non-fossil hydro-carbon based fuel, provided the $\mathrm{CO}_{2}$ emitted into the atmosphere is recaptured and recycled. One such scheme is power to gas $(\mathrm{P} 2 \mathrm{G})$, in which excess electricity generated by wind or solar power is converted and stored into hydro-carbon-based (solar) fuels. Naturally, for being $\mathrm{CO}_{2}$ neutral, the $\mathrm{CO}_{2}$ emitted by burning these solar fuels must be

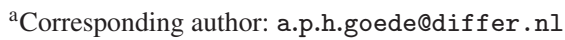

This is an Open Access article distributed under the terms of the Creative Commons Attribution License 4.0, which permits unrestricted use, distribution, and reproduction in any medium, provided the original work is properly cited. 
recaptured and reused, the so-called carbon capture and utilisation (CCU) scheme. Capture may be at source benefitting from high $\mathrm{CO}_{2}$ concentration, but eventually must include air capture to account for losses for the complete cycle to become $\mathrm{CO}_{2}$ neutral.

An important advantage of the P2G-CCU scheme is the use of existing infra structure for gas and oil storage, transport and distribution, whilst mobility technology remains hydro-carbon fuel based. The critical step in this scheme is the conversion of feedstock $\mathrm{CO}_{2}$ and $\mathrm{H}_{2} \mathrm{O}$ into syngas, which forms the starting point of chemical processing to obtain methane (Sabatier) or diesel (Fischer-Tropsch).

Currently, electrolysis and photo- or thermo-catalytic processes are used to split $\mathrm{H}_{2} \mathrm{O}$ and $\mathrm{CO}_{2}$ to produce $\mathrm{H}_{2}$ and $\mathrm{CO}$ respectively. Hydrogen production (without $\mathrm{CO}_{2}$ emission) is achieved by state of the art water electrolysis at an electric energy efficiency of about 65-75\%. The traditional alkali type systems are of considerable size, which limits application at MW level, notably off-shore. The energy density of Solid Oxygen Electrolysis Cells (SOEC) is higher $\left(1 \mathrm{~A} / \mathrm{cm}^{2}\right.$ at $1.5 \mathrm{~V}$, vs. $0.2 \mathrm{~A} / \mathrm{cm}^{2}$ for alkaline electrolyses) and its size correspondingly reduced. In addition, SOEC is based on gas rather than liquid, which eases pumping requirements and avoids bubble-clearing tanks. However, SOEC operates at $700-800^{\circ} \mathrm{C}$, which is not well suited to fast switching, because of thermal mechanical stress and corrosion at molecular interface layers. PEM (proton exchange membrane) electrolysers operate at a lower temperature of 50 to $80^{\circ} \mathrm{C}$ at similar energy density, but employ scarce and expensive materials such as Pt as a catalyst at the cathode.

\section{Plasmolysis of $\mathrm{CO}_{2}$}

Plasma processing (plasmolysis) overcomes these limitations by inherently higher power densities in the reactor of more than one order of magnitude and no scarce materials employed. A plasma reactor allows rapid switching to respond to intermittent demand. Plasmolysis is characterised by high energy efficiency and high production rate which can be scaled to industrial requirement.

The plasma (ionised gas) serves as a vehicle to ease conditions for splitting $\mathrm{CO}_{2}$ and $\mathrm{H}_{2} \mathrm{O}$. The plasma is produced by microwaves and accelerates the electrons, which vibrationally excite the $\mathrm{CO}_{2}$ and $\mathrm{H}_{2} \mathrm{O}$ molecules to a state conducive to dissociation into $\mathrm{CO}$ and $\mathrm{H}_{2}$ respectively. Such plasma is weakly ionised (only one in $10^{5}$ molecules is ionised) and relatively cold, similar to a fluorescent lamp. The vibrating molecules are not in thermal equilibrium with the gas temperature (translational motion) and hence no energy is wasted on heating the gas. The energy to produce the plasma is a relatively low factor in the energy balance. Most energy is channelled into vibrational excitation of $\mathrm{CO}_{2}$ and $\mathrm{H}_{2} \mathrm{O}$ molecules in order to lower the activation energy for splitting the molecule.

The energy efficiency $\eta$ for $\mathrm{CO}_{2}$ splitting is defined as the energy $\mathrm{E}_{\mathrm{CO}}$ required to produce one $\mathrm{CO}$ molecule output over the minimum enthalpy $\mathrm{H}$ required, $\eta=\mathrm{H} / \mathrm{E}_{\mathrm{CO}}$. The process proceeds in two steps; first $\mathrm{CO}_{2}$ is dissociated requiring minimum $5.5 \mathrm{eV}$ energy, second, the atomic oxygen radical produced reacts with another $\mathrm{CO}_{2}$ to produce $\mathrm{CO}$ at $0.3 \mathrm{eV}$. The net minimum enthalpy therefore is $\mathrm{H}=2.9 \mathrm{eV}$. The energy $\mathrm{E}_{\mathrm{v}}$ required per incoming $\mathrm{CO}_{2}$ molecule relates to energy $\mathrm{E}_{\mathrm{CO}}$ per $\mathrm{CO}$ molecule produced through the conversion factor $\alpha=\mathrm{E}_{\mathrm{v}} / \mathrm{E}_{\mathrm{CO}}$. The value of $\mathrm{E}_{\mathrm{v}}$ is amenable to measurement through $\mathrm{RF}$ power input $\mathrm{W}$ over input $\mathrm{CO}_{2}$ flow rate $\mathrm{F}$ according to:

$$
\eta=C F / W=\alpha H / E_{v} .
$$

Here $\mathrm{C}$ is a constant of numerical factors. The conversion factor $\alpha$ is determined experimentally from mass analysis of the exit gas stream.

\subsection{Physical model}

Equating production and loss, the steady state particle balance yields:

$$
2 v_{B}=n_{o}<\sigma_{i o n} v>r
$$


where $\mathrm{v}_{B}=\sqrt{ } 2 \mathrm{kT} \mathrm{T}_{\mathrm{e}} / \mathrm{m}_{\mathrm{i}}$ is the Bohm velocity of ions entering the plasma wall sheath, $\mathrm{n}_{\mathrm{o}}$ the neutral density, $\left\langle\sigma_{\text {ion }} v>\right.$ the ionisation rate averaged over electron velocity distribution and $\mathrm{r}$ the discharge radius. The ionisation rate is determined by the reduced electric field, see below.

From the energy balance equation an expression for electron density $\mathrm{n}_{\mathrm{e}}$ may be obtained:

$$
P_{R F}=n_{e} \Sigma_{i} n_{o}<\sigma_{i} v>\Delta \varepsilon_{i} V
$$

where $\sigma_{i}$ represents vibration, excitation and ionisation cross sections causing electron energy loss $\Delta \varepsilon_{i}$, $\mathrm{V}$ is the volume of the discharge. From the above expressions basic gas discharge parameters may be derived. Typically, plasma density is in the range $10^{12}-10^{13} \mathrm{~cm}^{-3}$, ionisation degree $10^{-5}$ and electron temperature in the range $1-10 \mathrm{eV}$.

The RF electric field, directed along the axis of the discharge, may be regarded stationary because all electron neutral collisions and plasma processes occur on much shorter time scale ( $\ll 1 \mathrm{~ns})$ compared with the RF oscillation period. A steady state electron drift establishes, striking a balance between acceleration in the E field and deceleration by neutral collision. The relevant parameter E/n, the reduced electric field, expresses the potential drop an electron experiences in between collisions. Through the randomisation process $\mathrm{E} / \mathrm{n}$ is proportional to the electron temperature.

Vibration excitation of $\mathrm{CO}_{2}$ in the asymmetric stretch mode shows a maximum cross section of $1.8 \times 10^{-16} \mathrm{~cm}^{2}$ at $0.4 \mathrm{eV}$ electron energy [3]. Establishing this energy over the mean free path corresponds to a value $\mathrm{E} / \mathrm{n}=1.4 \times 10^{-16} \mathrm{Vcm}^{2}$. According to [4.5] this is the most efficient route to $\mathrm{CO}_{2}$ dissociation, whilst direct electron impact or dissociative attachment is less energy efficient. The vibration state needs to be maintained for vibration energy to build-up and surmount the activation barrier. This is the case at low translational gas temperature where V-T relaxation is reduced [4]. Low translational temperature may be achieved by supersonic expansion of the gas flow [6].

\subsection{Experimental results}

Initial $\mathrm{CO}_{2}$ plasmolysis experiments are conducted at the IGVP Stuttgart RF plasma facility, consisting of a cylindrical RF cavity ( $\mathrm{TE}_{010}$ mode at $915 \mathrm{MHz}$ ) in which a $\mathrm{CO}_{2}$ plasma is created [7]. Gas composition measurements are based on quadrupole mass spectrometry and optical emission spectroscopy (OES) of $\mathrm{CO}$. The $\mathrm{CO}_{2}$ gas flow is injected tangentially at the entrance of the reaction chamber, which consists of a cylindrical quartz flow tube (length $124 \mathrm{~mm}$ and dia. $26 \mathrm{~mm}$ ) placed on axis of the RF cavity. Gas pressure is measured at the entrance and the exit of the reaction chamber.

Three types of discharge have been investigated at constant input $\mathrm{CO}_{2}$ gas flow rate $11.1 \mathrm{slm}$ : Type I gas expansion nozzle dia. $10 \mathrm{~mm}$ is placed at the entrance of the RF cavity expanding the gas into the discharge. Typical gas pressure $1 \mathrm{mbar}$ is characterised by high $\mathrm{E} / \mathrm{n} \sim 100 \times 10^{-16} \mathrm{Vcm}^{2}$. Typical energy efficiency $\eta=15 \%$ is found constant with power and particle conversion $\alpha$ increases linearly with power. OES shows dominance of $3^{\text {rd }}$ and $4^{\text {th }}$ positive $\mathrm{CO}$ bands, increasing linear with power. Type II gas expansion nozzle is placed at the exit of the RF cavity freely expanding the converted gas stream. The high pressure 200 mbar gas discharge is characterised by low E/n $10^{-16} \mathrm{Vcm}^{2}$. Typical energy efficiency $\eta=35 \%$ decreases with power. Particle conversion increases with power from $10 \%$ upward. The OES is dominated by the CO Angström and CO Triplet system whilst the Swan system reveals Carbon formation. In accord with Eq. (2), the discharge diameter has become contracted.

Type III exit nozzle diameter has been increased in steps from $5 \mathrm{~mm}$, to $10 \mathrm{~mm}$, to $26 \mathrm{~mm}$ dia. This type of discharge is characterised by an intermediate reduced electric field regime $\mathrm{E} / \mathrm{n} \sim 10 \times 10^{-16} \mathrm{Vcm}^{2}$.

In order to increase energy efficiency, Type II high gas flow experiments at $75 \mathrm{slm}$ and $3 \mathrm{~kW}$ power were conducted, reaching record energy efficiency $>50 \%$ at low conversion fraction $10 \%$. Power density is typically $45.5 \mathrm{~W} / \mathrm{cm}^{3}$. At $200 \mathrm{mbar}$ the $\mathrm{CO}$ output flow becomes $20 \mathrm{~A} / \mathrm{cm}^{2}$ equivalent.

The E field has been calculated by Comsol Multiphysics simulation program [8]. The vacuum field strength on axis of the cavity is $E[V / c m]=4.965 \sqrt{ } P_{R F}[W]$. Since the RF frequency is much lower 


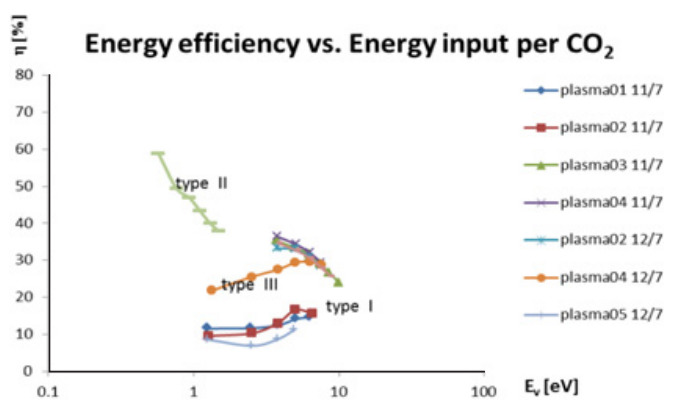

Figure 1. Energy efficiency $\eta$ for $\mathrm{CO}$ production increases by lowering energy $\mathrm{E}_{\mathrm{v}}$ per incoming molecule $\mathrm{CO}_{2}$.
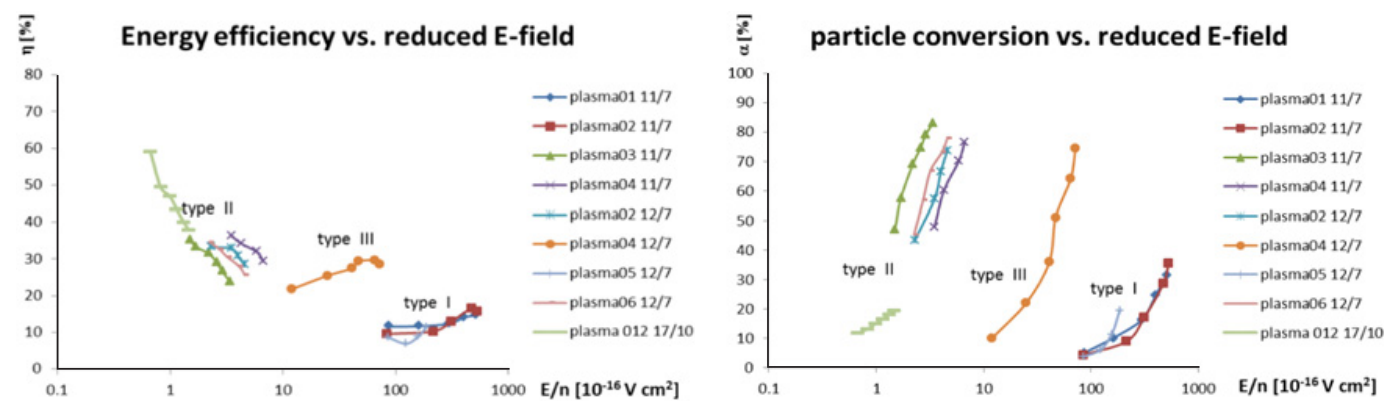

Figure 2. Left: lowering the reduced electric field $\mathrm{E} / \mathrm{n}$ is key to increased energy efficiency $\eta$ but, Right: goes at the expense of the conversion fraction $\alpha$, consistent with $\mathrm{E}_{\mathrm{v}}$ values below the dissociation energy.

than the cut-off plasma frequency, power is absorbed by collisionality of the plasma decreasing the $\mathrm{E}$ field by the complex refractive index. This aspect has been ignored in the E estimate.

The particle density has been estimated by assuming $\mathrm{M}=1$ flow at the nozzle, i.e. flow velocity equals sound velocity scaling as $\sqrt{ } \mathrm{T}$. As a consequence, at constant flow the gas density scales $\mathrm{n} \sim 1 / \sqrt{ } \mathrm{T}$. The pressure is seen to increase linear with power; hence gas temperature $\mathrm{T}$ increases with power.

Results are collated in Figs. 1 and 2. The trend suggests higher efficiencies may be obtained at lower $\mathrm{E} / \mathrm{n}$ values. Here, conflicting requirements on sustaining the gas discharge requiring $\mathrm{T}_{\mathrm{e}}>3 \mathrm{eV}$ and low E/n values requiring $\mathrm{T}_{\mathrm{e}}<1 \mathrm{eV}$ are encountered, suggesting decoupling of requirements necessary. Energy efficiency goes up with decreasing energy $\mathrm{E}_{\mathrm{v}}$ per incoming $\mathrm{CO}_{2}$ molecule, whilst particle conversion goes down. This result is consistent with Eq. (1), and is tantamount to saying that available energy per input $\mathrm{CO}_{2}$ molecule needs to be concentrated on one in many $\mathrm{CO}_{2}$ molecules in order to accumulate sufficient energy to attain the net $2.9 \mathrm{eV}$ dissociation energy.

\section{Conclusions}

The reduced electric field has been identified as the key parameter in explaining and improving the energy efficiency measured for the various type of plasmolysis discharge studied. Energy efficiency obtained is similar to electrolysis, but offers advantages in energy density and switching in response to intermittent supply with no use of scarce material. A simple model explains the inverse relation between energy efficiency and particle conversion factor and relates the input RF power to $\mathrm{CO}_{2}$ gas density, plasma dimension and ionisation degree, allowing $100 \mathrm{~kW}$ reactor up-scaling parameters to be specified, taking due account of reduced electric field requirements. 


\section{$3^{\text {rd }}$ European Energy Conference}

\section{References}

[1] Z. Boldiš, Europhys News 44/4, 16 (2013)

[2] S Weitemeyer et al., this conference

[3] Y. Itikawa, J. Phys. Chem. Ref. Data, 31, 749 (2002)

[4] V. D. Rusanov, A. A. Fridman, and G. V. Sholin, Usp. Fiz. Nauk. 134, 185 (1981)

[5] A.A. Fridman, Plasma Chemistry, Cambridge (2008), ISBN-13 978-0-511-39857-5

[6] R.I. Azizov et al.; Sov. Phys. Dokl. 28(7) 567 (1983)

[7] M. Leins et al., $31^{\text {th }}$ Int. Conf. Phen. in Ionized Gases, Granada (2013)

[8] M. Leins, Thesis Ch 2.3.2 (2010), Universität Stuttgart 